Supporting Information for

\title{
Long-Chain Branched Polypentenamer Rubber: Topological Impact on Tensile Properties, Chain Dynamics, and Strain-Induced Crystallization
}

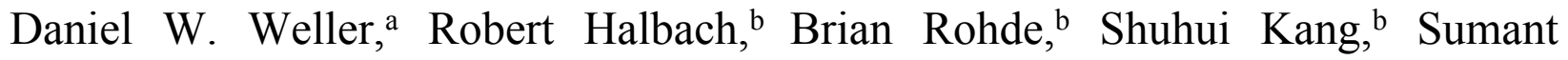
Dwivedi, ${ }^{a}$ Kyle D. Mehringer, ${ }^{a}$ Rahul Shankar, ${ }^{a}$ Robson F. Storey, ${ }^{a}$ Sarah E. Morgan, ${ }^{a}$ Alexander V. Zabula, ${ }^{b}$ Xiaodan Gu ${ }^{a^{*}}$, Carlos R. López-Barró ${ }^{\mathrm{b}^{*}}$

a School of Polymer Science and Engineering, The University of Southern Mississippi, Hattiesburg, Mississippi 39406, United States

${ }^{\mathrm{b}}$ ExxonMobil Chemical Company, 5200 Bayway Drive, Baytown, Texas 77520, United States

*Corresponding Authors: Email: xiaodangu@usm.edu, carlos.r.lopez-barron@exxonmobil.com 


\section{PPR Vulcanization Recipe}

Table S1 Recipe for PPR vulcanization.

\begin{tabular}{|l|c|}
\hline Ingredient & phr $^{\mathbf{a}}$ \\
\hline PPR & 100 \\
\hline Zinc stearate & b \\
\hline DPG $^{\mathrm{c}}$ & 0.5 \\
\hline CBS $^{\mathrm{d}}$ & 0.2 \\
\hline Sulfur & 0.2 \\
\hline
\end{tabular}

${ }^{\mathrm{a}}$ phr: parts per hundred rubber.

${ }^{\mathrm{b}}$ Technical grade (Sigma-Aldrich)

'DPG: diphenyl guanidine (98\%, TCI)

${ }^{\mathrm{d}}$ CBS: N-cyclohexyl-2-benzothiazole sulfonamide ( $>98 \%$, TCI)

eSuperfine sulfur ( $99.5 \%$, Akrochem)

\section{Branched and Linear DSC Scans}
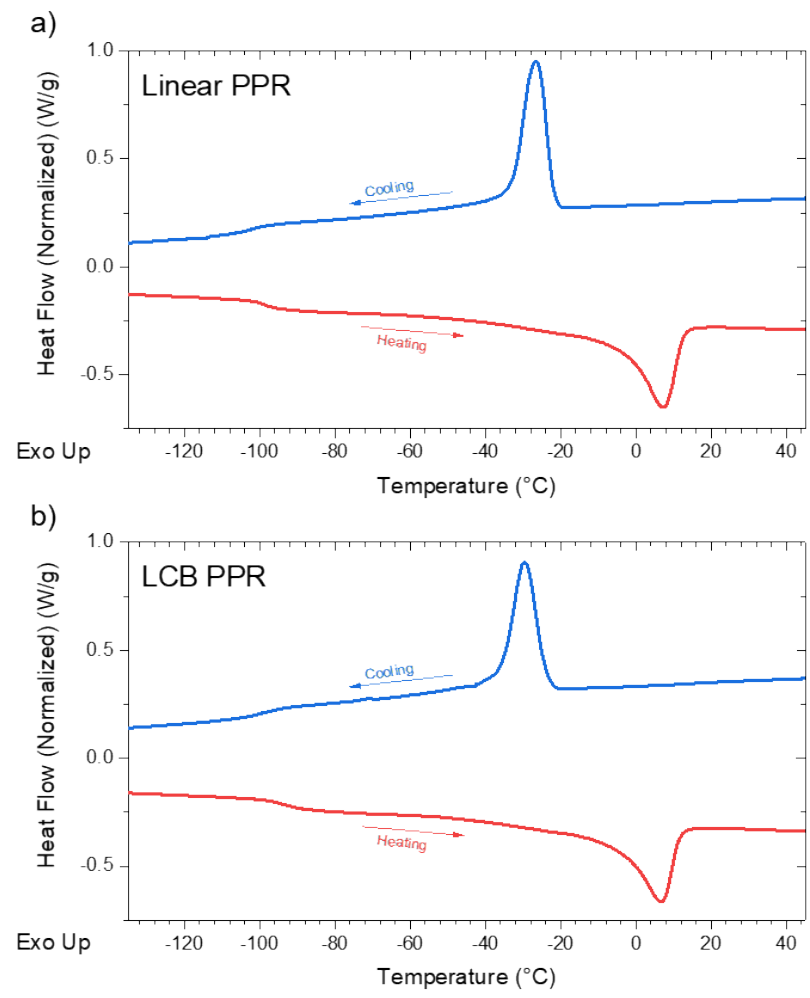

Figure S1: DSC Scans of a) Linear PPR and b) Branched PPR. 


\section{Crystallinity Calculation and Peak Assignment}

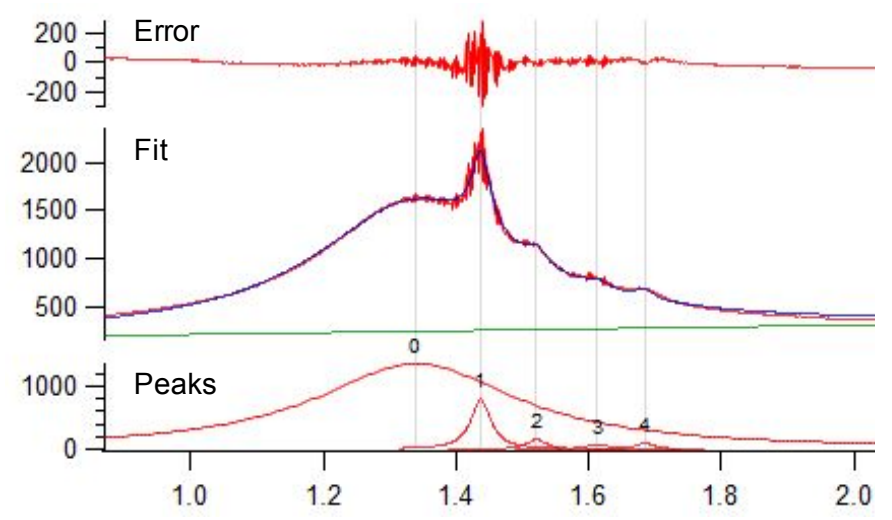

Figure S2: Igor curve fitting software. 1 amorphous (labeled as peak 0) and 4 crystalline peaks (labeled as peak 1 4) were used to fit data. The red line is the data, blue line is the model.

$\%$ Crystallinity $=100 \times \frac{\sum \text { Peak areas } 1,2,3,4}{\sum \text { Pea } \text { area } 0,1,2,3,4}$

\section{Alignment Factor Calculation}

a)

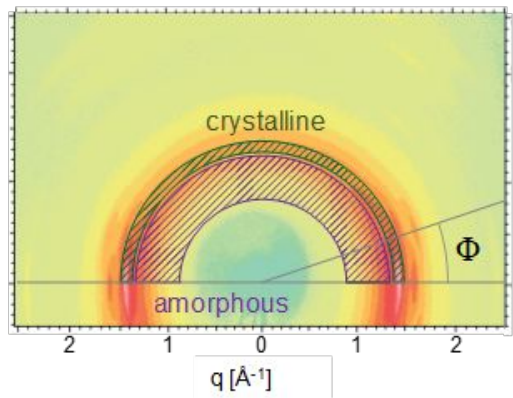

b)

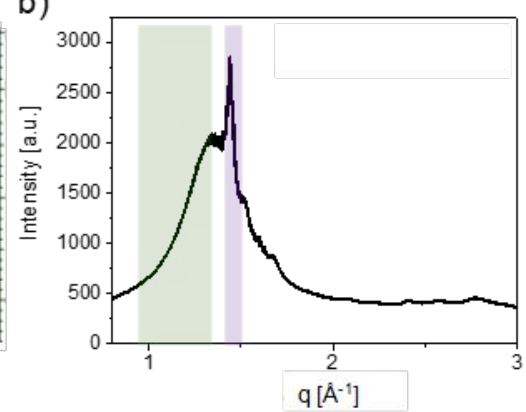

c)

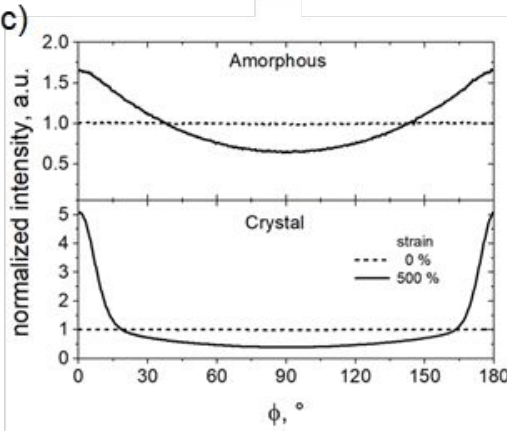

Figure S3: Method for extracting alignment information from WAXS data. a) Amorphous and crystalline $q$ regions on $2 D$ scattering plot which were plotted vs. azimuthal angle. b) Amorphous and crystalline $q$ regions used for azimuthal angle plot shown on $1 D$ plot. c) Azimuthal angle vs. intensity plot for amorphous and crystalline $q$ ranges showing alignment in stretch direction. 
Figure S3 shows the q range and methods used for the alignment calculations. Notice that half of the amorphous halo was not considered to avoid complication from crystalline peak overlap. When intensity in these q ranges are plotted versus azimuthal angle $(\Phi)$ a plot like Figure $7 \mathrm{c}$ is obtained which gives an indication of the alignment present in the plot. Higher intensity near $0^{\circ}$ and $180^{\circ}$ comes from chains aligned with stretch. The intensity around $90^{\circ}$ represents chains aligned perpendicular to stretch.

\section{Branching Index Calculation from GPC Data}
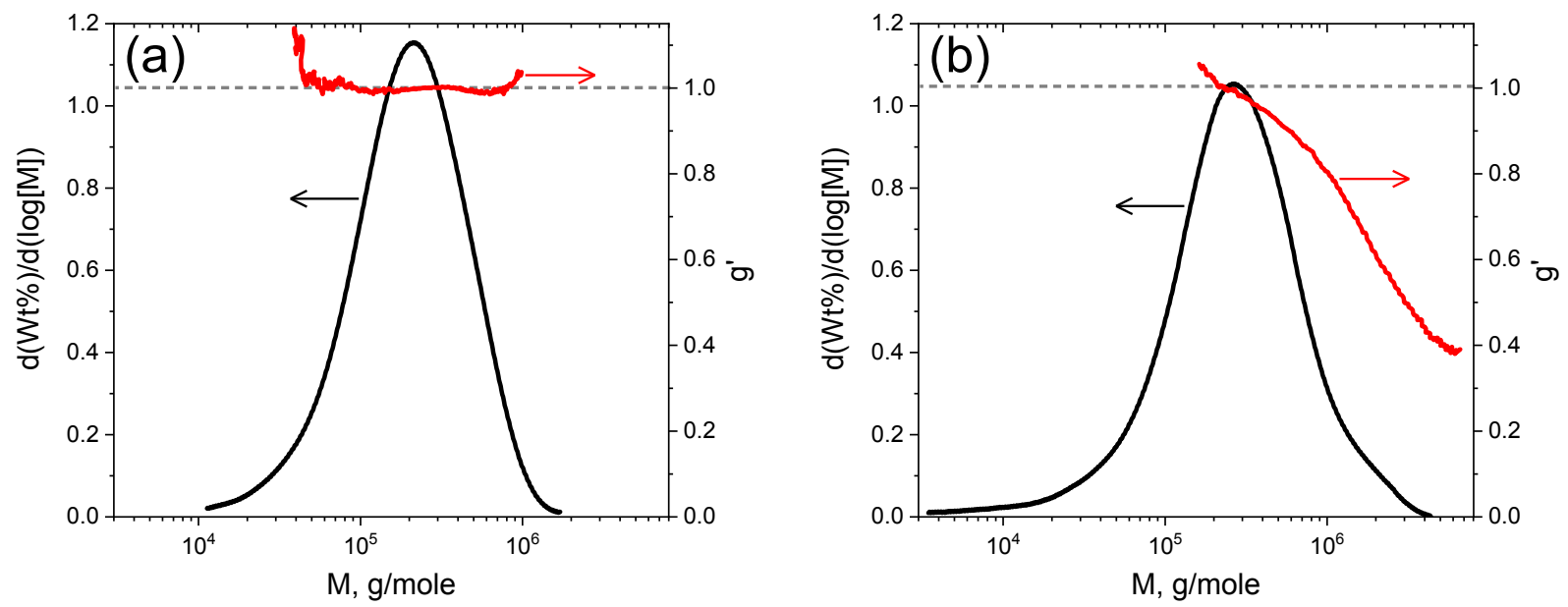

Figure S4: Molecular weight density and branching index ( $g$ ') versus molecular weight $(M)$ for linear and branched CP polymers. a) Linear CPR branching index is M-independent around 1. b) Branched CPR has an average branching index at 0.81 and decreases with increasing $M$, demonstrating branching is occurring.

\section{AFM Domain Size Analysis}
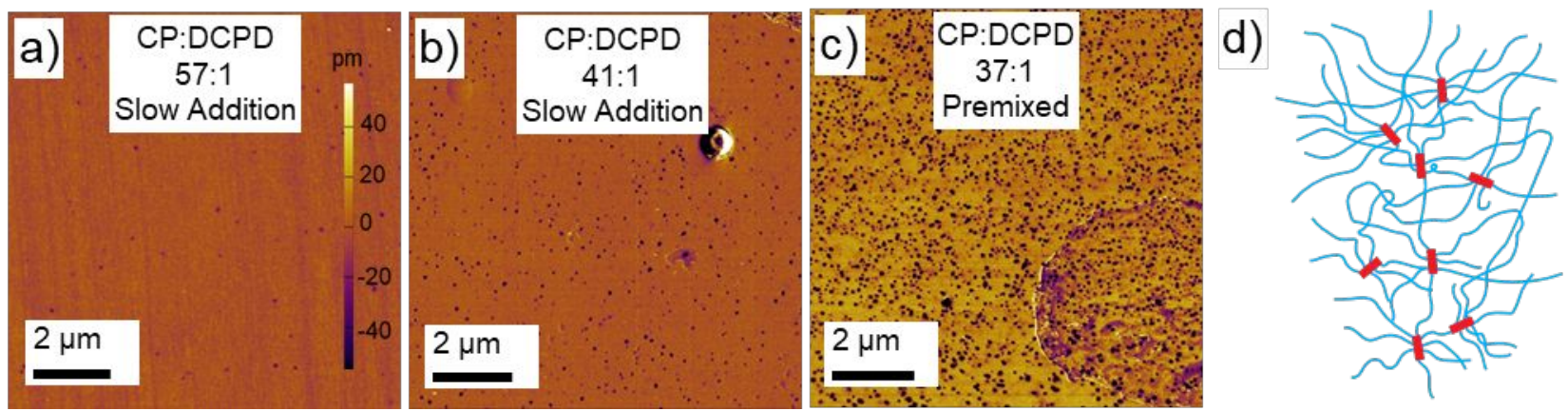

Figure S5: AFM height images showing increased surface pitting with higher levels of DCPD. a) CP to DCPD monomer ratio of 57:1 contains minimal amounts of depressions. Scale bar applicable to all micrographs. b) Higher CP to DCPD monomer ratio of 41:1 ratio shows increased size and abundance of depressions. c) CP to DCPD ratio of 37:1 where all DCPD was premixed with CP at start of reaction rather than slowly added. d) Proposed topology of the long chain branched CP:DCPD copolymerization. 
Hard DCPD domains (red) provide the majority of branching sites, all of which are interconnected through linear CP chains.

Atomic force microscopy (AFM) performed on cured PPR revealed nano-segregation occurring in the in branched samples (Figure S5). The size and number of these nanodomains were influenced by both the amount of DCPD added, and the rate at which DCPD was added. The synthetic procedure and characterization of the PPRs for each DCPD level can be found in the next section. Because the nanodomain prevalence increases with increasing DCPD loading level, combined with the fact that DCPD's $\mathrm{T}_{\mathrm{g}}$ is much higher than $\mathrm{CP}\left(163^{\circ} \mathrm{C}\right)$, we believe the dark dots in the micrographs are glassy DCPD-rich hard domains embedded in the CP-rich soft matrix. We attribute the nanostructure observed in the LCB PPRs to the much larger ROMP reactivity of DCPD compared to that of CP. This results in the formation of blocky DCPDrich chain segments being formed as soon as DCPD is delivered to the reactive solution. When this is done slowly during the course of the reaction, the local DCPD concentration can diffuse relatively fast and, thus, a more uniformly branched architecture is produced. However, formation of some concentrated DCPD domains cannot be avoided, and a few hard-glassy domains are formed (Figures S5a and S5b). If all the DCPD is added initially, this monomer will have less chance to diffuse and react homogeneously with CP, and more glassy domains will be formed, as seen in Figure S5c.

We postulate that these domains act as branching hubs from where multiple branches originate, as illustrated in Figure S5d. The hyperbranched topology is formed when DCPD monomers propagated for existing branches and form a satellite branching hub. Interestingly, no crosslinking events occur during the reaction, and these polymers can be melt processed before vulcanization. Moreover, the hard DCPD domains may act as very well dispersed nanofillers with very effective reinforcement effect, which is likely contributing to branched PPR's increased tensile properties, obfuscating the effect branching would have on its own.

\section{Synthesis and characterization of CP:DCPD copolymers used in AFM Study}

CP:DCPD 41:1 Slow Addition. The catalyst was formed in situ by adding solid \{4$\left.\left(\mathrm{PhCH}_{2}\right) \mathrm{C}_{6} \mathrm{H}_{4} \mathrm{O}\right\}_{2} \mathrm{AlCl}(865 \mathrm{mg}, 2.02 \mathrm{mmol})$ to a solution of $\mathrm{WCl}_{6}(400 \mathrm{mg}, 1.01 \mathrm{mmol})$ in toluene $(20 \mathrm{~mL})$. After stirring for one hour at ambient conditions, the resulting mixture was added to a solution containing cyclopentene (first comonomer, $275 \mathrm{~g}, 4.035 \mathrm{~mol}$ ), triethylaluminum (activator, $230 \mathrm{mg}, 2.02 \mathrm{mmol}$ ), and toluene $(2200 \mathrm{~mL})$ at $0{ }^{\circ} \mathrm{C}$. A solution of DCPD (second comonomer) (3.6 g, $\left.27.3 \mathrm{mmol}\right)$ in toluene (15 $\mathrm{mL}$ ) was slowly added to the reaction mixture over 35 minutes under intense mechanical stirring. After additional stirring for $20 \mathrm{~min}$, a solution of 2,6-di-tert-butyl-4-methylphenol (1.00 g, $4.48 \mathrm{mmol})$ in 100 $\mathrm{mL}$ of ethanol/toluene mixture (1:4, v:v, respectively) was added. The obtained mixture was then added to methanol $(1.5 \mathrm{~L})$. The precipitated polymer was washed with methanol $(500 \mathrm{~mL} \times 3)$ and dried under vacuum at $55^{\circ} \mathrm{C}$ for 4 hours to give $98 \mathrm{~g}$ of the final product. Yield: $35 \%$. DCPD incorporation: $2.5 \mathrm{~mol} \%$ $(\mathrm{CP}: \mathrm{DCPD}=41: 1)$. The details of characterization are summarized in Table 3.

CP:DCPD 37:1 Premixed. In contrast to CP:DCPD 41:1 Slow Addition, both monomers were combined together before adding the catalyst. The catalyst was formed in situ by adding solid $\{4-$ $\left(\mathrm{PhCH}_{2}\right) \mathrm{C}_{6} \mathrm{H}_{4} \mathrm{O}_{2} \mathrm{AlCl}(865 \mathrm{mg}, 2.02 \mathrm{mmol})$ to a solution of $\mathrm{WCl}_{6}(400 \mathrm{mg}, 1.01 \mathrm{mmol})$ in toluene $(20 \mathrm{~mL})$. After stirring for one hour at ambient conditions, the resulting mixture was added to a solution containing 
cyclopentene (first comonomer, $275 \mathrm{~g}, 4.035 \mathrm{~mol}$ ), DCPD (second comonomer, $3.6 \mathrm{~g}, 27.3 \mathrm{mmol}$, triethylaluminum (activator, $230 \mathrm{mg}, 2.02 \mathrm{mmol}$ ), and toluene $\left(1200 \mathrm{~mL}\right.$ ) at $0{ }^{\circ} \mathrm{C}$. After 1 hour, a solution of 2,6-di-tert-butyl-4-methylphenol $(1.00 \mathrm{~g}, 4.48 \mathrm{mmol})$ in $100 \mathrm{~mL}$ of ethanol/toluene mixture $(1: 4, \mathrm{v}: \mathrm{v}$, respectively) was added. The obtained mixture was added to ethanol $(1.5 \mathrm{~L})$. The precipitated polymer was washed with ethanol $(500 \mathrm{~mL} \times 3)$ and dried under vacuum at $55^{\circ} \mathrm{C}$ for 4 hours to give $77 \mathrm{~g}$ of the product. Yield: $28 \%$. DCPD incorporation: $2.7 \mathrm{~mol} \%(\mathrm{CP}: \mathrm{DCPD}=37: 1)$. The details of characterization are summarized in Table 3.

Table S2: Material properties for PPRs used in AFM study

\begin{tabular}{|l|l|l|l|}
\hline & $57: 1$ Slow Addition & $41: 1$ Slow Addition & $37: 1$ Premixed \\
\hline cis/trans mole ratio & $19 / 81$ & $20 / 80$ & $23 / 77$ \\
\hline $\mathrm{cC} 5 / \mathrm{DCPD}$ & $57 / 1$ & $41 / 1$ & $37 / 1$ \\
\hline $\mathrm{Mw}, \mathrm{kg} / \mathrm{mol}$ & 475 & 614 & 611 \\
\hline $\mathrm{Mw} / \mathrm{Mn}$ & 1.98 & 1.70 & 1.75 \\
\hline $\mathrm{Tg},{ }^{\circ} \mathrm{C}$ & -93.4 & na & na \\
\hline $\mathrm{Tm}$ (peak), ${ }^{\circ} \mathrm{C}$ & 6.62 & na & na \\
\hline Tc (onset), ${ }^{\circ} \mathrm{C}$ & -23.7 & na & na \\
\hline
\end{tabular}

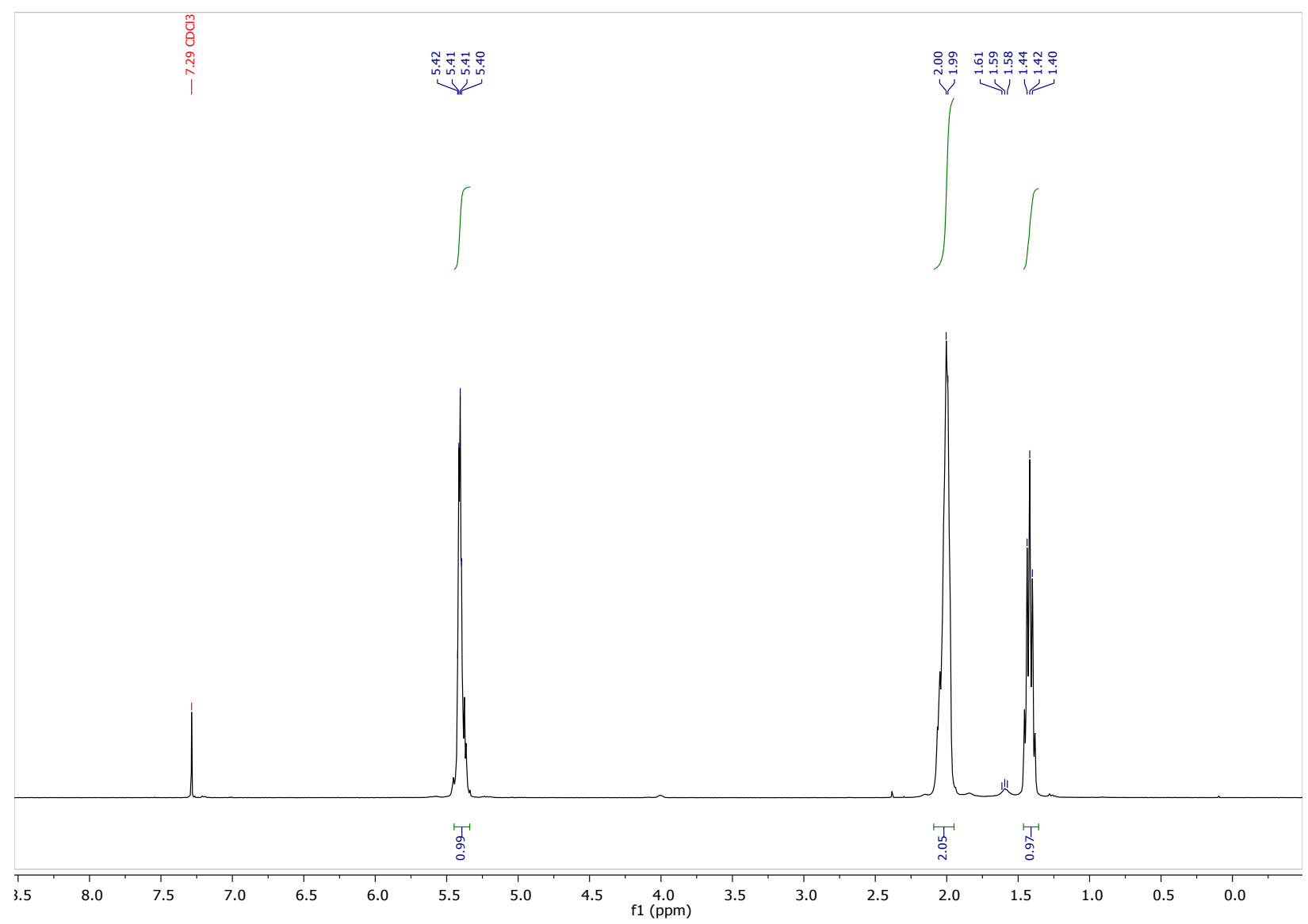

Figure S6: ${ }^{1} H$ NMR spectrum for linear PPR (in $\mathrm{CDCl}_{3}$ ). 


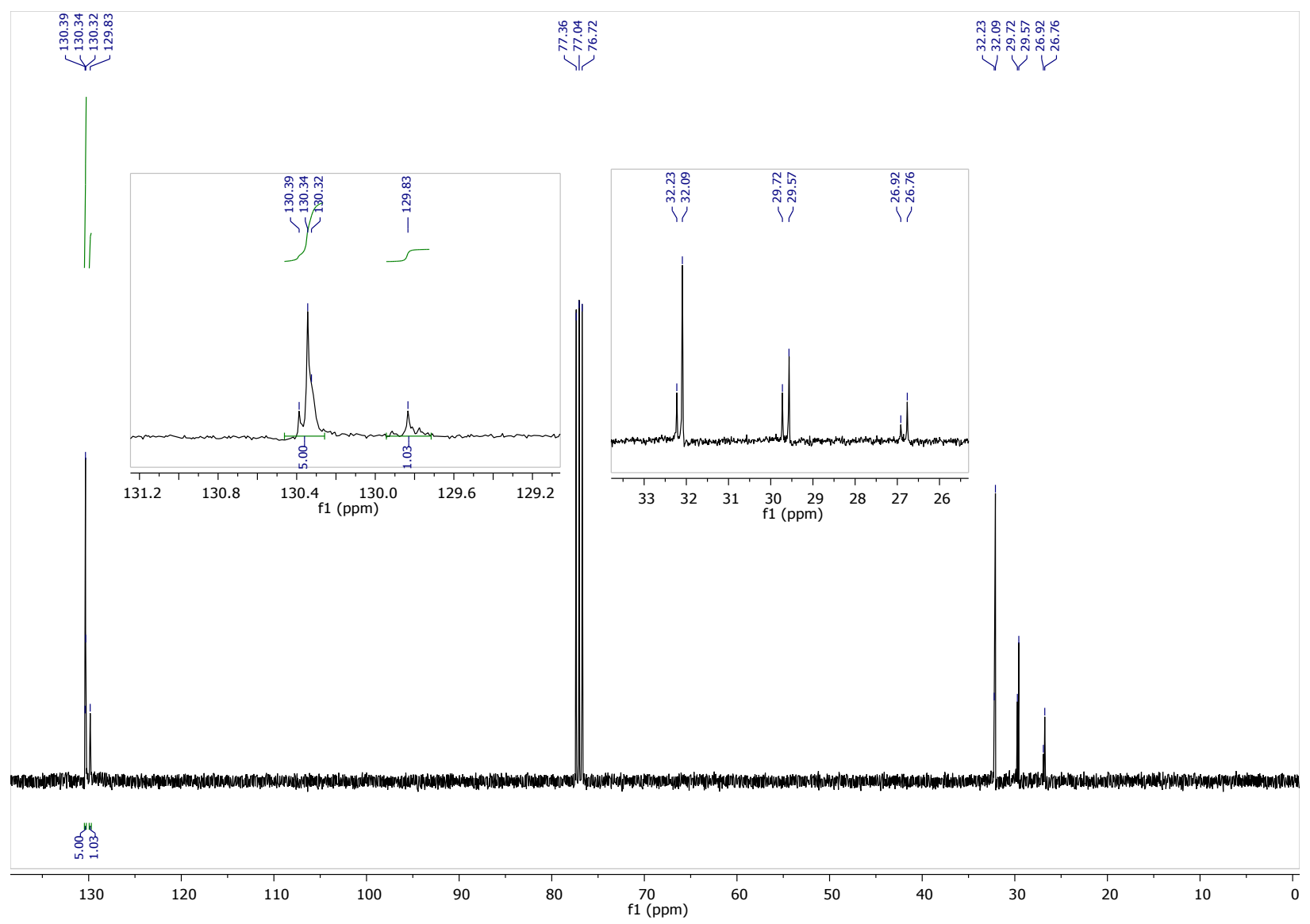

Figure S7: ${ }^{13} C$ NMR spectrum for linear PPR (in $\mathrm{CDCl}_{3}$ ). 


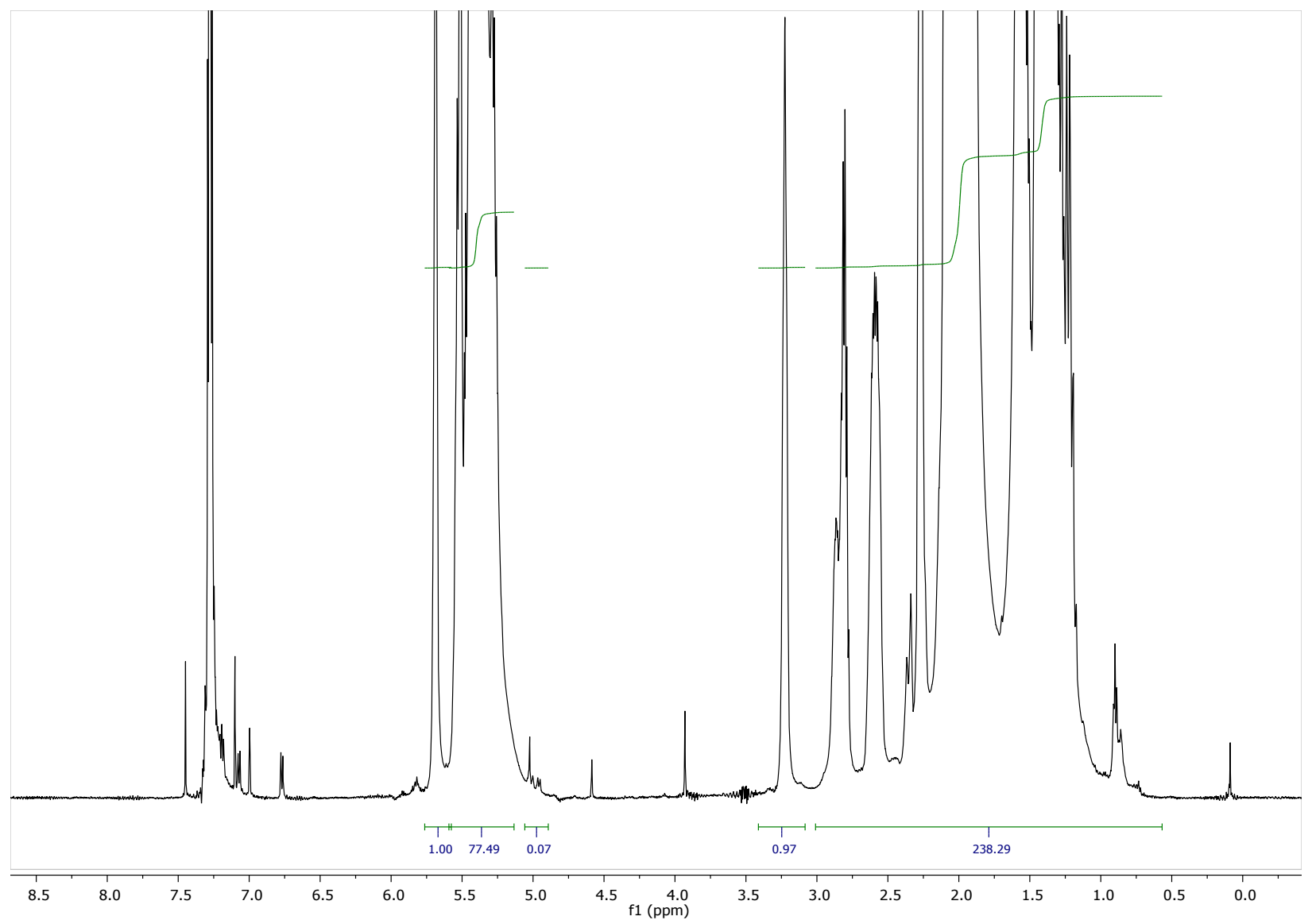

Figure S8: ${ }^{1} \mathrm{H} N M R$ spectrum for $L C B P P R$ (in $\left.C D C l_{3}\right)$. 


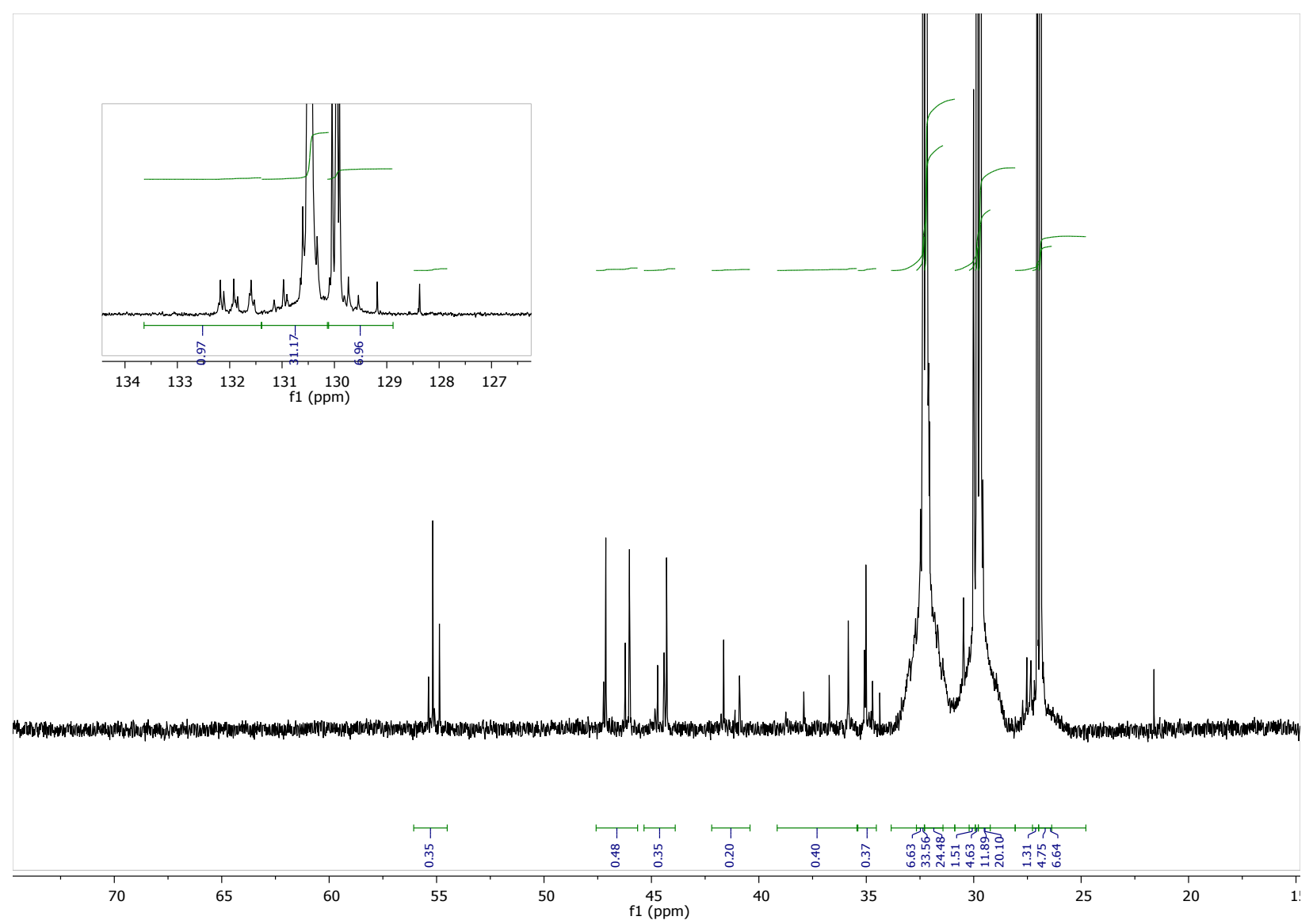

Figure S9: ${ }^{13} C N M R$ spectrum for $L C B P P R$ (in $C D C l_{3}$ ). 


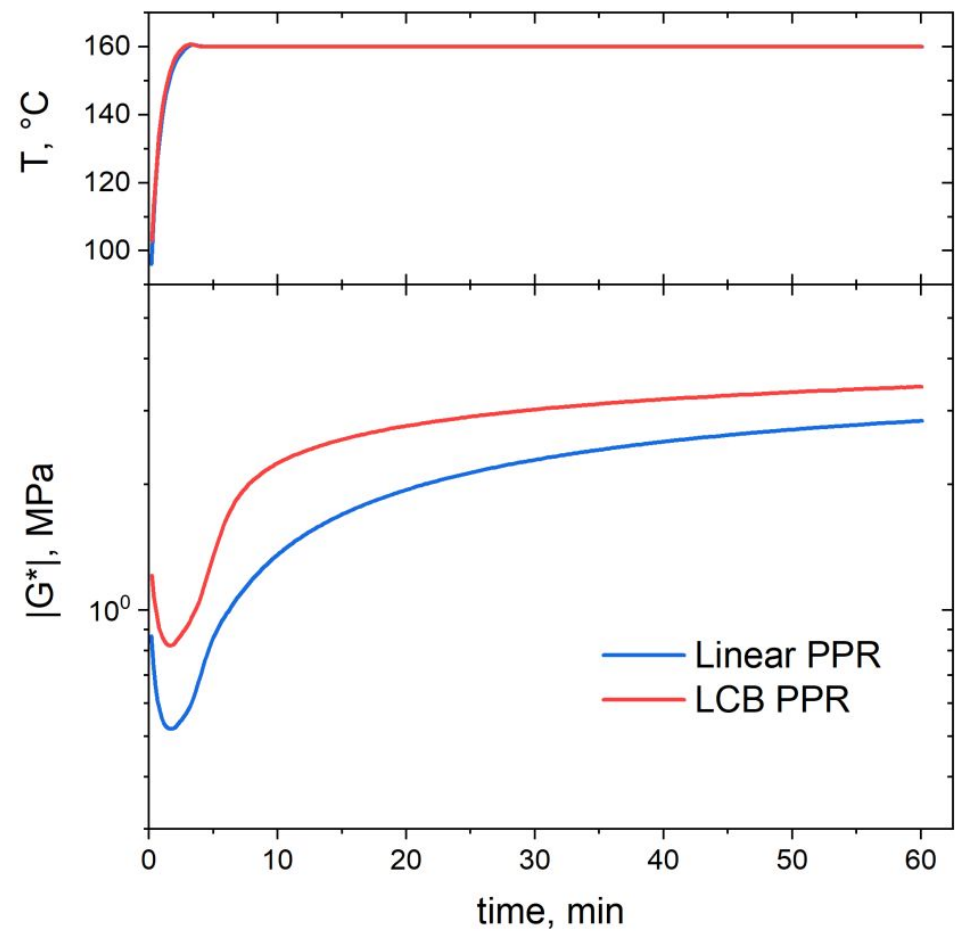

Figure S10. Cure kinetic curves for linear and LCB PPRs measured in the rheometer ARES-G2 using a frequency of $1 \mathrm{~Hz}$ and strain amplitude of $0.1 \%$. Samples are loaded at $80{ }^{\circ} \mathrm{C}$ in $8 \mathrm{~mm}$ serrated parallel plates geometry, after which the temperature is increased to $160^{\circ} \mathrm{C}$. 